\title{
Camouflage and Display for Soft Machines
}

\section{Citation}

Morin, Stephen A., Robert F. Shepherd, Sen Wai Kwok, Adam A. Stokes, Alex Nemiroski, and George M. Whitesides. 2012. Camouflage and Display for Soft Machines. Science 337, no. 6096: 828-832.

\section{Published Version}

doi:10.1126/science. 1222149

\section{Permanent link}

http://nrs.harvard.edu/urn-3:HUL.InstRepos:11933749

\section{Terms of Use}

This article was downloaded from Harvard University's DASH repository, and is made available under the terms and conditions applicable to Open Access Policy Articles, as set forth at http:// nrs.harvard.edu/urn-3:HUL.InstRepos:dash.current.terms-of-use\#OAP

\section{Share Your Story}

The Harvard community has made this article openly available.

Please share how this access benefits you. Submit a story.

Accessibility 


\title{
Title: Camouflage and Display for Soft Machines
}

Authors: Stephen A. Morin ${ }^{1}$, Robert F. Shepherd ${ }^{1}$, Sen Wai Kwok ${ }^{1}$, Adam A. Stokes ${ }^{1}$, Alex Nemiroski ${ }^{1}$, and George M. Whitesides ${ }^{1,2^{*}}$

\author{
Affiliations: \\ ${ }^{1}$ Department of Chemistry and Chemical Biology, Harvard University, 12 Oxford Street, \\ Cambridge, MA 02138. \\ ${ }^{2}$ Wyss Institute for Biologically Inspired Engineering, Harvard University, 60 Oxford Street, \\ Cambridge, MA 02138. \\ *Correspondence to: gwhitesides@gmwgroup.harvard.edu.
}

Abstract: Synthetic systems cannot easily mimic the color-changing abilities of animals such as cephalopods. Soft machines, machines fabricated from soft polymers and flexible reinforcing sheets, are rapidly increasing in functionality. This manuscript describes simple microfluidic networks that can change the color, contrast, pattern, apparent shape, luminescence, and surface temperature of soft machines for camouflage and display. The color of these microfluidic networks can be changed simultaneously in the visible and infrared - a capability that organisms do not have. These strategies begin to imitate the functions, although not the anatomies, of color-changing animals.

Main Text: Cephalopods (e.g., squid and cuttlefish) have amazing control over their appearance (color, contrast, pattern, and shape) $(1,2)$. These animals use dynamic body patterns for disguise, for protection, and for warning. Other animals (e.g., chameleons and many insects) can also actively change their coloration for camouflage or display $(3,4)$. Yet others (e.g., jellyfish and fireflies) use bioluminescence to communicate (5). The color-changing capabilities of these animals have not been replicated using soft synthetic systems, but such systems could enhance the function of certain machines (e.g., robots or prosthetics).

This paper describes our initial approaches to change the color, contrast, pattern, apparent shape, luminescence, and infrared (IR) emission (that is, surface temperature) of soft machines 
fabricated from elastomers and flexible reinforcing sheets (6-8) by changing the color and pattern of microfluidic networks. These systems are first steps toward imitating the functions, although not the anatomies, of cephalopods $(9,10)$ and other color-changing animals (4). These animals typically change color using specialized cells, such as chromatophores or iridophores $(4$, 9, 10), not simple microchannels. The near-perfect matching of environments used by colorchanging organisms with highly developed nervous systems is not required for camouflage to be effective.

Nature offers countless examples of camouflage and display $(3,11,12)$. While specific demonstrations of camouflage vary among species, the strategies used have common themes: background matching, disruptive coloration, and disguise $(3,11,12)$. In background matching, animals use colors and patterns similar to their habitats $(3,11-13)$; an example is counter/obliterative shading (e.g., rabbits with white underbellies and brown backs) $(11,12)$. Disruptive coloration breaks up the silhouette of an animal using contrasting patterns that do not follow its shape (3). Disguise is a strategy where an organism adopts the shape and color of objects or animals in the environment (e.g., stick insects and phasmida) (3). Display strategies are used for communication, mating, and hunting, and can be just as critical for survival as camouflage (3). Bioluminescence is commonly used for display in low- or no-light conditions $(5,14)$; when lighting is strong, animals sometimes advertise themselves by combining vibrant contrasting colors with distinct shapes (15-17). For example, jellyfish use bioluminescence to warn would be predators (5) and peacocks use bright plumage to attract mates (18).

While most strategies of camouflage and display $(3,12)$ emphasize the visible spectrum that most animals (especially mammals) can see (19-21), there are animals that can see (or otherwise sense) ultraviolet or IR light, and that use these spectral regions for signaling or 
hunting. For example, most birds, many arthropods, and some fish have ultraviolet vision (22) and many snakes, such as pit vipers, can sense IR light using specialized organs (23). Semiconductor technology has expanded our ability to see into the IR (24), and we explored techniques for camouflage or display in the IR.

Our system combines microfluidics, pattern, and color to provide both camouflage/display and movement of the soft machines. Camouflage or display results from pumping colored or temperature-controlled fluids through a network of microfluidic channels; mechanical actuation results from pneumatic pressurization and inflation of an independent network of microchannels (pneu-nets) embedded in highly extensible elastomers $(6,7)$. The microfluidic networks used for camouflage/display are contained in thin silicone (Ecoflex ${ }^{\circledR}, 1-2$ mm thick) sheets referred to as color layers (Fig. 1 and fig. S1). While there are technologies, such as electrowetting (25) and electrofluidics (26), that use microfluidics to tune color, they rely on electric fields to move fluid, and are not immediately compatible with our compressed air power source.

The color layers are easily fabricated (27), can cover large areas, and can transport a variety of fluids (both liquids and gases) using similar controls (28). The liquids can be colored with dyes or pigments and heated/cooled to change the color of the microfluidic networks in the visible and IR spectrum; the possible combinations of colorants at different temperatures provide greater flexibility in spectral tailoring than can be achieved using other technologies:

thermoelectrics can change the IR signature, and electronic displays can change the visible color, but neither technology has control over both IR and visible coloration. Further, once filled, the color layers require no power, have low requirements for volume of fluid $\left(\sim 30 \mu \mathrm{L} / \mathrm{cm}^{2}\right.$ of surface), and are lightweight (130 mg/ $\mathrm{cm}^{2}$ of surface). We used closely packed microfluidic 
channels (Fig. 1), or combined microfluidic channels with wider ( $\mathrm{mm}$ to $\mathrm{cm}$ ) channels (fig. S1D), to create features that are indistinguishable from continuous color in the far field.

Ecoflex $^{\circledR}$, unlike the materials used in commercially available flexible electronic displays, has a Young's modulus compatible with the flexible bodies and motions of soft machines, and is also mechanically robust and inexpensive. The translucency of Ecoflex ${ }^{\circledR}$ also helped the machines blend in with their surroundings.

We used a soft robotic quadrupedal "walker" (7) to demonstrate that the color layers are light and flexible enough to be compatible with a mobile, stretchy soft machine. We took advantage of the regular and symmetrical shape of the quadruped to illustrate camouflage strategies that can break up recognizable silhouettes.

We attached the color layers to the top of soft quadrupeds, fabricated as described previously (Fig. 1) (6, 7). Using a pumping rate of $2.25 \mathrm{~mL} / \mathrm{min}$ and neglecting the time necessary to fill the tether, a change in color required 30 seconds (Fig. 1D; color-change was also monitored with reflectance spectroscopy, fig. S6). The weight and flexibility of the color layer did not significantly slow the robot's locomotion (the velocity was 0.6 times the velocity of the same robot without the color layer; Fig. 1E) (7).

Figure 2 illustrates the operation of camouflage in a rock bed (movie S1), and a leafcovered concrete slab using two different color layers (Fig. 1 and fig. S1D) filled with two different sets of colored solutions. In all demonstrations, the colored solutions were prepared manually by the operator. Both designs created large, disjointed patches of contrasting brightness similar to the environment, but not to the shape of the robot; this disjuncture in shape (disruptive coloration (3)) helped conceal the robot. Simple image analysis performed on the camouflaged and un-camouflaged robot (Fig. 2B, Fig. 3, fig. S4, and fig. S5) demonstrated that 
the brightness of the camouflaged robot was significantly closer to the brightness (within the deviation defined by RMS contrast; fig. S4) of the environment than that of the un-camouflaged robot $(27,29)$. Additionally, we found the similarity in pattern between the background and the camouflaged robot to be favorable (fig. S5). This brightness/contrast resemblance and similarity in pattern was important to this demonstration.

We also investigated the camouflage of soft robots (using background matching $(3,11$ 13)) in a patterned environment similar to a tiled floor (Fig. 2, E to F, and fig. S2) by creating a color layer with periodic microfluidic channels filled with colors matched to that environment. Although not perfectly camouflaged, the colored robots are less obvious and demonstrate the potential of this system. Dynamic camouflage would be useful for applications where soft machines must do their job without standing out (e.g., soft robots performing maintenance operations).

Patterns and contrasting coloration schemes that make the soft machine stand out against a background (display), while less technically demanding than camouflage, are just as important and useful (an example would be to aid location and recovery of soft machines from poorly lit or cluttered environments). We changed the camouflage/display modes of the color layers simply by changing the fluid within them (Fig. 1C, bottom panels). This ability of a single color layer to switch between modes of coloration is an advantage. We further illustrate display by filling dense, shape-matching color layers (fig. S1B) with fluorescent (Fig. 2, G to H, and movie S2), black and white (Fig. 2J), or chemiluminescent (Fig. 2I and movie S3) solutions that revealed the location of the robots in light or dark.

We also demonstrated camouflage and display in the IR spectrum (Fig. 4 and movie S4), using warm $\left(70{ }^{\circ} \mathrm{C}\right)$ or cool $\left(2^{\circ} \mathrm{C}\right)$ colored solutions. Pumping these temperature-controlled 
solutions through the color layer created contrasting IR color patterns that make the system stand out against its thermal environment. This strategy makes it possible simultaneously to change the visible and IR colors. As an illustration, we camouflaged a color layer within the visible spectrum while simultaneously displaying it in the IR spectrum (Fig. 4, F to G). At a pumping rate of $2.25 \mathrm{~mL} / \mathrm{min}$ we achieved IR color change in 80 seconds (movie S4). The rate of IR color change was limited by the thermal conductivity of Ecoflex ${ }^{\circledR}\left(0.16 \mathrm{Wm}^{-1} \mathrm{~K}^{-1}(27)\right)$ and not the pumping rate.

Simple systems can approach some of the functionality of dynamic coloration that many animals use to control their appearance (4). Microfluidic networks that make up a color layer that is independent of other functions can effectively, albeit crudely when compared to animals, camouflage or display soft machines by altering their apparent shape, color, contrast, luminescence, temperature, and pattern. The ability of color layers simultaneously to change color in the visible and the IR is a capability not used by organisms, and not easily replicated using other technologies: animals are limited in their ability to control their temperature; soft machines fabricated in silicone elastomers are not.

These devices confirm that microfluidic channels can serve multiple functions in soft machines: actuation, camouflage, display, fluid transport, and temperature regulation. Combining different functional microfluidic systems simplifies the design and increases the functionality of soft machines. Complex/specialized microfluidic networks can take the place of these relatively simple color layers to bring more capabilities (e.g., reagent handling and storage) to soft machines.

We believe the simple design, fabrication, and operation of these camouflage/display techniques for soft machines make these systems accessible and potentially useful to many 
different scientific fields. Biologists studying camouflage/display could use specially designed soft machines to observe how dynamic color, temperature, and pattern influence animal behavior (e.g., predator/prey relationships) $(13,30)$. In anatomy, our devices could simulate fluid vessels and muscle motion for realistic modeling or training. While we have focused on soft robots, our display systems can also interface with hard robots and present new opportunities for modifying the appearance of these devices. These applications are approachable using tethered microfluidic systems like those demonstrated here, but some applications will demand more technically advanced autonomous systems. The challenges associated with autonomy will be addressed initially using larger-bodied machines, or robots that can carry power sources, pumps, electronics, and fluids. We imagine such systems could eventually include feedback sensors for active camouflage.

\section{References and Notes:}

1. R. T. Hanlon, Curr. Biol. 17, R400-R404 (2007). http://dx.doi.org/10.1016/j.cub.2007.03.034.

2. $\quad$ R. T. Hanlon et al., Philos. Trans. R. Soc. B-Biol. Sci. 364, 429-437 (2009).

3. H. B. Cott, Adaptive Coloration in Animals, (Methuen, London, 1957).

4. D. Stuart-Fox, A. Moussalli, Philos. Trans. R. Soc. B-Biol. Sci. 364, $463-470$ (2009).

5. J. W. Hastings, J. Mol. Evol. 19, 309-321 (1983).

6. F. Ilievski, A.D. Mazzeo, R.F. Shepherd, X. Chin, G.M. Whitesides, Angew. Chem.-Int. Edit. 50, 1890-1895 (2011).

7. R. F. Shepherd et al., Proc. Natl. Acad. Sci. U. S. A. 108, 20400-20403 (2011).

8. R. V. Martinez, C. R. Fish, X. Chen, G. M. Whitesides, Adv. Funct. Mater. 22, 13761384 (2012).

9. L. M. Mathger, R. T. Hanlon, Cell Tissue Res. 329, 179-186 (2007).

10. R. L. Sutherland, L.M. Mathger, R.T. Hanlon, A.M. Urbas, M.O. Stone, J. Opt. Soc. Am. A-Opt. Image Sci. Vis. 25, 588-599 (2008).

11. G. H. Thayer, A. H. Thayer, Concealing-Coloration in the Animal Kingdom : An Exposition of the Laws of Disguise Through Color and Pattern: Being a Summary of Abbott H. Thayer's Discoveries, (Macmillan, New York, 1909). 
12. M. Stevens, S. Merilaita, Philos. Trans. R. Soc. B-Biol. Sci. 364, 423-427 (2009).

13. S. Merilaita, J. Lind, Proc. R. Soc. Lond. Ser. B-Biol. Sci. 272, 665-670 (2005).

14. T. Wilson, J. W. Hastings, Annu. Rev. Cell Dev. Bi. 14, 197-230 (1998).

15. K. V. Langridge, Anim. Behav. 77, 847-856 (2009).

16. M. D. Norman, J. Finn, T. Tregenza, Proc. R. Soc. Lond. Ser. B-Biol. Sci. 266, 1347-1349 (1999).

17. E. Scholes, Biol. J. Linn. Soc. 94, 491-504 (2008).

18. M. Petrie, T. Halliday, C. Sanders, Anim. Behav. 41, 323-331 (1991).

19. G. H. Jacobs, Biol. Rev. Camb. Philos. 68, 413-471 (1993).

20. T. H. Goldsmith, Q. Rev. Biol. 65, 281-322 (1990).

21. D. G. Luo, W. W. S. Yue, P. Ala-Laurila, K. W. Yau, Science 332, 1307-1312 (2011).

22. D. M. Hunt, S. E. Wilkie, J. K. Bowmaker, S. Poopalasundaram, Cell. Mol. Life Sci. 58, 1583-1598 (2001).

23. R. C. Goris, J. Herpetol. 45, 2-14 (2011).

24. A. Rogalski, Prog. Quantum Electron. 27, 59-210 (2003).

25. R. A. Hayes, B. J. Feenstra, Nature 425, 383-385 (2003).

26. J. Heikenfeld et al., Nat. Photonics 3, 292-296 (2009).

27. Information on materials and methods is available on Science Online.

28. G. M. Whitesides, Nature 442, 368-373 (2006).

29. J. A. Endler, Biol. J. Linn. Soc. 41, 315-352 (1990).

30. I. C. Cuthill et al., Nature 434, 72-74 (2005).

Acknowledgments: The development of biomimetic camouflage strategies was supported by the Department of Energy under award number DE-FG02-00ER45852. Soft machine development was supported by the Defense Advanced Research Projects Agency under award number W911NF-11-1-0094. Patent applications "Flexible Robotic Actuators" and "Soft Robotic Actuators" have been filed but not yet published.

\section{Supplementary Materials:}

SOM Text

Materials and Methods

Figures S1-S6 
References (31-35)

Movies S1-S4 


\section{Figures:}

Fig. 1. Design and operation of a color layer. (A) Schematic of a disruptive color layer comprising two channels. The dotted black line indicates the final shape. (B) Cross-sectional schematic of the region indicated by the dotted red line in A. (C) Various patterns of coloration generated by filling (or not filling) the channels of a completed color layer with solutions of dye (top four images) and pigment dispersions (bottom two). The dotted line in the top left panel shows the outline of the quadrupedal soft robot. (D) Reversible coloration. This color layer design shows the operation of the system, and is not intended to camouflage/display the machine. Colored aqueous solutions are pumped using a syringe. The pumping rate was $2.25 \mathrm{~mL} / \mathrm{min}$ (channel volume $=0.75 \mathrm{~mL}) .(\mathbf{E})$ Despite the added weight of a filled color layer, a soft quadruped moves at $\sim 40 \mathrm{~m} / \mathrm{h}$ (0.6 times the velocity of the same device without the color layer). The major ruler divisions are $1 \mathrm{~cm}$. 

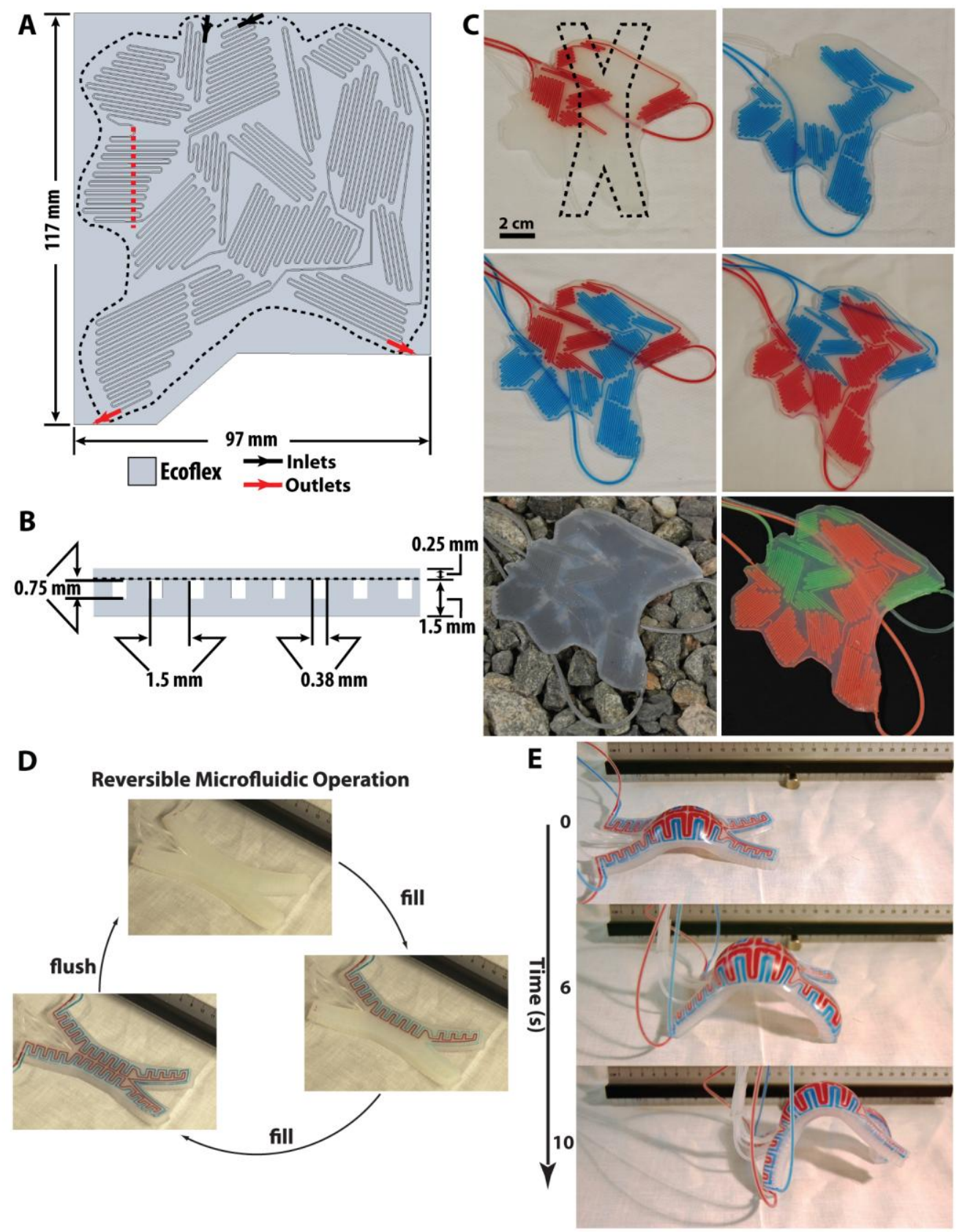
Fig. 2. Camouflage and display coloration. (A) Sequential images of a soft robot walking onto a rock bed where it is camouflaged by pumping colored pigment dispersions through the microfluidic channels in the color layer. The number at the bottom left corner of each frame is the time (seconds). The tether is intentionally left visible to contrast the colors used against a white background. (B) Close-up images of the robot un-camouflaged (left) and camouflaged (right). (C and D) Images of a soft robot walking onto a leaf-covered concrete slab where it is camouflaged. (E) Close-up image of an un-camouflaged (top) soft robot in an artificial, manmade environment and the same soft robot camouflaged (bottom). (F) The robot from $\mathbf{E}$ in five different regions adopting coloration for background matching. One position of the robot from $\mathbf{E}$ is marked with a dashed box; fig. S3 shows all five. (G) Fluorescent orange robot displayed on a rock bed. (H) Robot from $\mathbf{G}$ displayed on a leaf-covered rock bed. (I) Robot glowing in the dark using chemiluminescence (inset: the same robot photographed in the light). (J) Robots displayed in contrasting black and white on the pattern from F. All quadrupeds were $13 \mathrm{~cm}$ long (Fig. 1). 


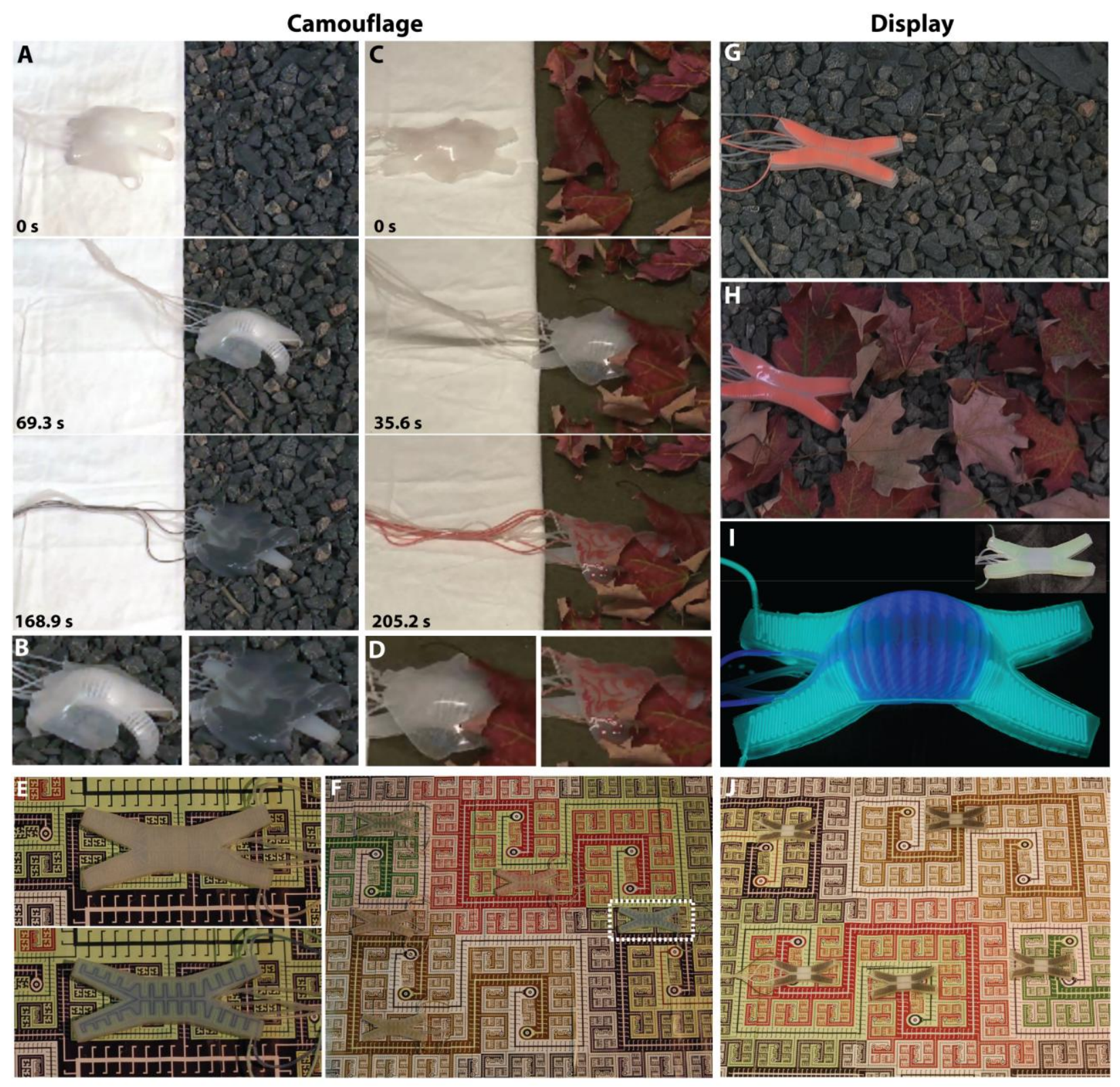


Fig. 3. Stacked histograms showing the distribution of gray scale intensities (brightness) generated by image analysis of a rocky environment (background), a rocky environment with an un-camouflaged soft robot, and a camouflaged soft robot. Insets are the analyzed images. 


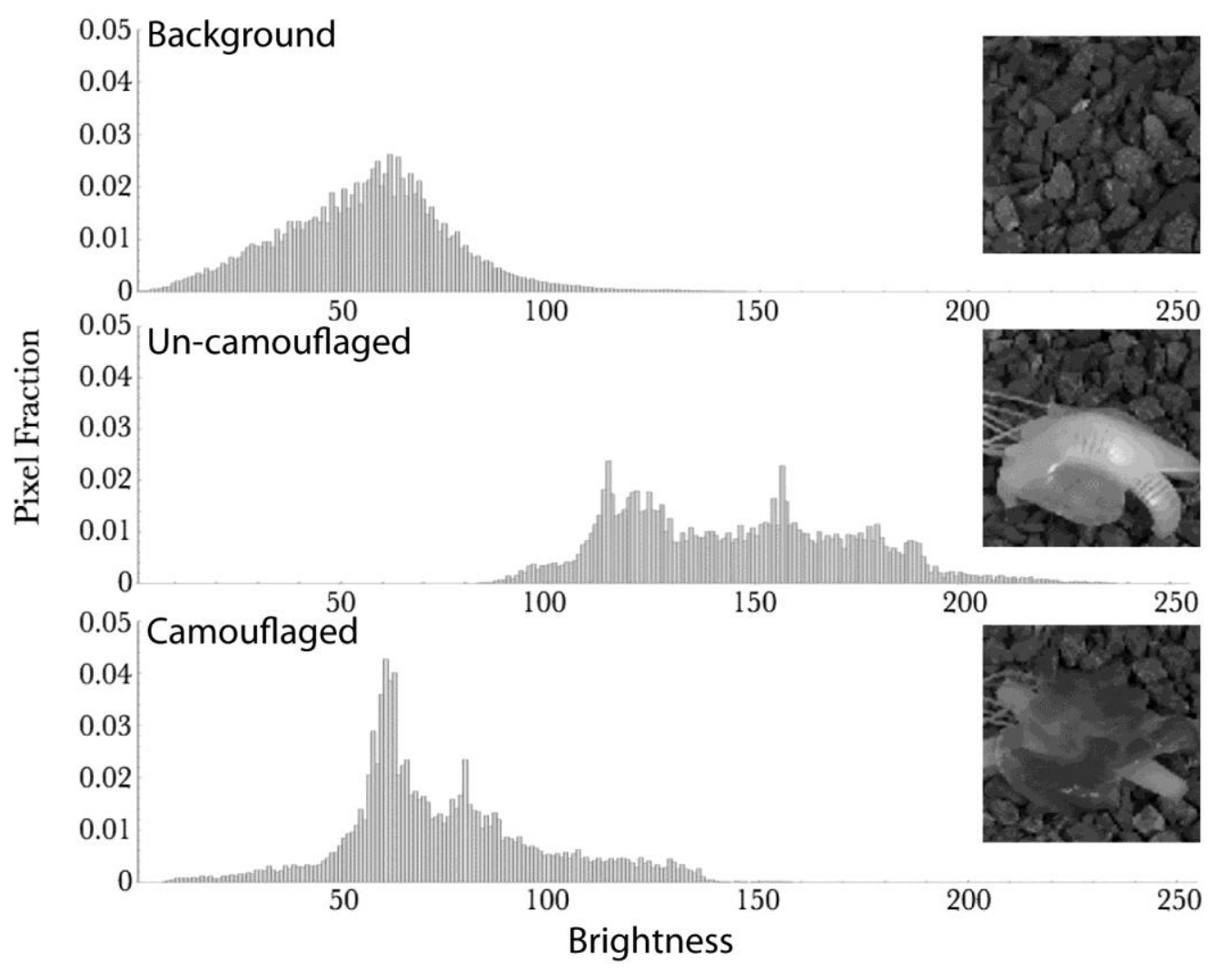


Fig. 4. Visible (left column) and IR (right column) images showing the simultaneous color change of two different color layers in both spectral regions. The IR images for the first color layer (A to $\mathbf{E}$ ) share the temperature scale from $\mathbf{A}$ and the IR images for the second ( $\mathbf{F}$ and $\mathbf{G}$ ) share the temperature scale from $\mathbf{G}$. Panels $\mathbf{A}$ to $\mathbf{E}$ share the scale bar in $\mathbf{A}$; panels $\mathbf{F}$ and $\mathbf{G}$ share the scale bar in $\mathbf{F}$. The number in the bottom left corner is the time (seconds) of each frame. (A) The color layer is empty. (B) Warm $\left(70^{\circ} \mathrm{C}\right)$ blue dye and cool $\left(2{ }^{\circ} \mathrm{C}\right)$ red dye are pumped through the color layer (flow rate $=2.25 \mathrm{~mL} / \mathrm{min}$ ). $(\mathbf{C}$ to $\mathbf{D})$ The dyes are exchanged with $2{ }^{\circ} \mathrm{C}$ water and $70{ }^{\circ} \mathrm{C}$ water. (E) Room temperature $\left(22^{\circ} \mathrm{C}\right)$ water is pumped into both channels. (F) An unfilled color layer is seen in the visible, but camouflaged in the IR. (G) The color layer from $\mathbf{F}$ is camouflaged in the visible using two shades of grey pigment dispersions. One dispersion is at $70{ }^{\circ} \mathrm{C}$ and the other at $2{ }^{\circ} \mathrm{C}$. 
Visible

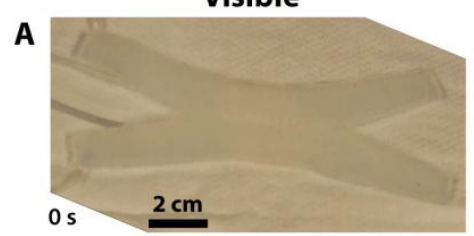

B

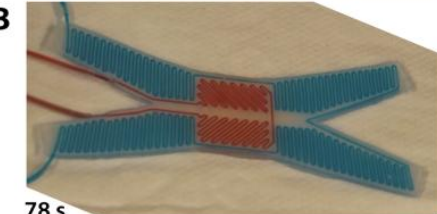

C
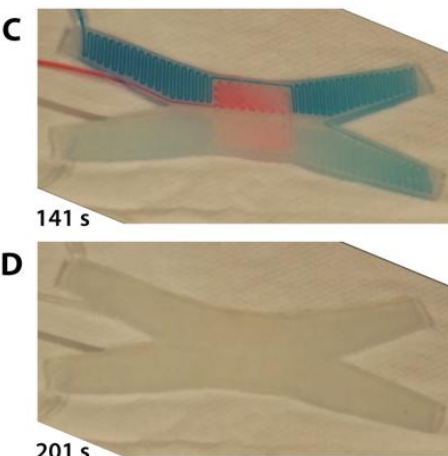

E
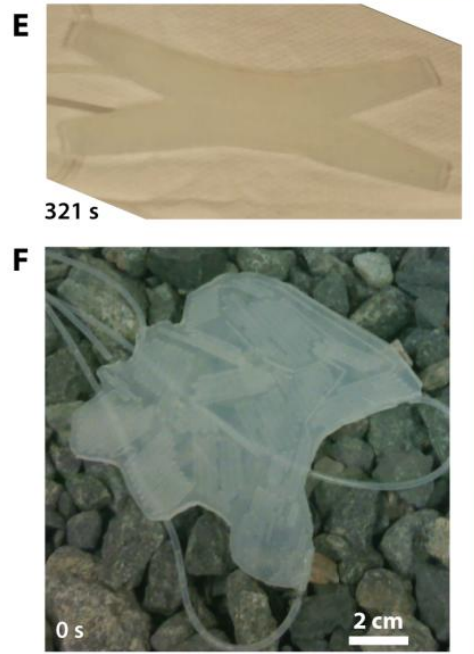

G

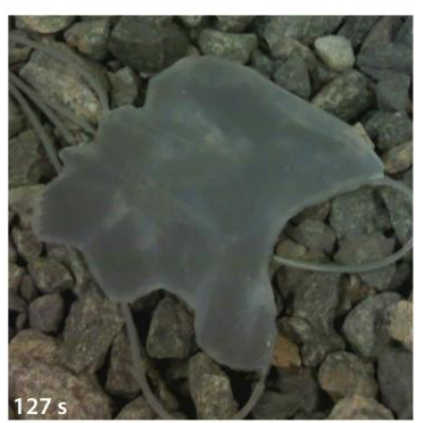

IR
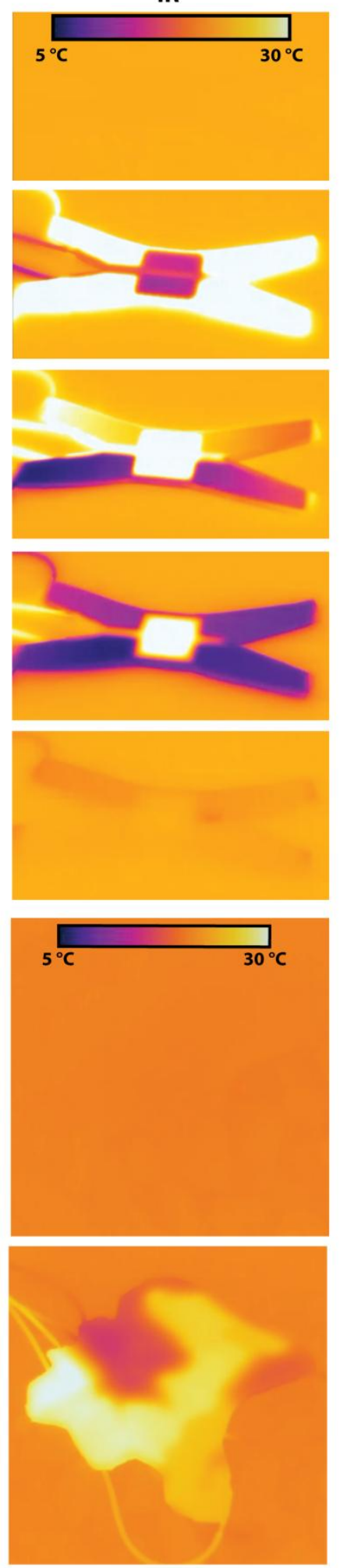